pearance of these intermediates shows that the folding can be considered to occur in three steps, each associated with the appearance of a further disulphide bridge. The first step is the formation of one-disulphide intermediates which are in rapid equilibrium with each other. The rate-limiting step in their disappearance is the formation of two-disulphide intermediates which are rapidly converted by the formation of the third bridge into the biologically active inhibitor.

Those intermediates with one disulphide bridge are more closely investigated, largely because results concerning the two-disulphide intermediates are less reproducible. The most abundant species of single disulphide intermediates has a disulphide bridge between cys residues at positions 30 and 51 in the amino acid sequence, and constitutes about half the population of single-disulphide molecules. The next most abundant species has a disulphide bridge between cys-5 and cys-30, and constitutes about one quarter of the population. Two minor species, involving bridges between cys30 and cys-55 and between cys-5 and cys-51, are also detectable.

Returning to the analogy with biological evolution, the importance of the predominating species with a bridge at cys-30 to cys-51 is that this bridge constitutes a 'living fossil' in the sense that it survives to appear in the native, folded structure. Contemporary theories of protein folding lay great emphasis on the importance of such 'living fossils'. It is widely held that local arrangements of the backbone of the protein, such as $\alpha$ helix and $\beta$-pleated sheet, form early during the folding but survive to appear in the native structure because of their intrinsic stability. Since none of the other detectable disulphide bridging arrangements appears in the native structure, it is interesting to consider whether the 30-51 bridge fits in with this notion. Creighton points out that in the native structure cys-30 occurs in the middle of a $\beta$ pleated sheet region and cys-51 in the middle of an $\alpha$-helical region. Since the initial formation of helix and pleated sheet regions is generally supposed to be followed by mutual association, the early appearance of a bridge between cys-30 and cys-51 is consistent with the early appearance of the helix and pleated sheet features in which they occur.

A further bonus is that the early formation of the $\beta$-pleated sheet region would account for the correct threading of the backbone. Correct threading is a particular problem for trypsin inhibitor because to arrive at the native conformation the backbone must be passed through the loop formed by the 30-51 bridge. Without the initial formation of the $\beta$-pleated sheet, it would be hard to see the factors which select for correctly threaded molecules.

Because of the emphasis on disulphide bridging it might seem paradoxical that the usefulnes of Creighton's results depends on cys residues not having a special role in the folding of proteins. In fact it is crucial that disulphide bridges form only when the conformation of the molecule, determined by other factors, will permit. Hence the trapping of the disulphide intermediates is intended merely to be a device for freezing the folding process at any moment in time and it is hoped that the disulphide bridges which form are an effect, never a cause, of the conformational preferences of the intermediates. In order to satisfy this ideal it is necessary that an interaction between cys residues is no stronger than non-covalent interactions between other sidechains at the time of folding. Although the covalent nature of the disulphide bridge would seem to make this an unrealistic ideal, the disulphide bond is in fact a labile association whose stability is determined by the reducing potential of the environment. It is therefore very pertinent that, as pointed out by Creighton, trypsin inhibitor is synthesised biologically in conditions where the reducing potential actually disfavours disulphide bridge formation. Evidence summarised by Creighton would seem to suggest that cys residues are cunningly placed by nature so as to form stabilising disulphide bridges in an extracellular oxidising environment, but that they have no relevance to the normal folding process.

The most general conclusion of this work is that a non-random mixture of conformations can be formed early during the folding process, and that some of the features of the most sbundant intermediates may survive to appear in the native conformation. The most important result specific to trypsin inhibitor is that the missing link from the early stages of folding is represented by the disulphide bridge cvs-30 to cys-51.

\section{Meteorites and rabbits}

from David $W$. Hughes

THE collection of meteorites in the Australian Museum has increased enormously in the past few years, and a large portion of the new material has resulted from the activities and interests of a group of rabbit trappers who criss-cross the Nullabor Plain on motor cycles. In fact, out of 36 meteorites recovered from the plain 24 were found by the members of one family, the Carlisles. The added involvement of the Kalgoorlie School of
Mines and the Western Australian Museum has increased the rate of detection of meteorites from 16 per decade, which remained constant between 1897 and 1966 , to around 80 per decade since 1966.

Until the latest count (Rec. Austr. Mus., 29, 169; 1974), reported by Mason (Smithsonian Institution, Washington DC), irons formed the most numerous group of Australian meteorites. This is in marked contrast to general world statistics, in which stones predominate. A possible explanation for this is that meteoritic iron may have provided raw material for swords and ploughshares. Iron meteorites would therefore have been consumed rapidly once a native people had acquired the facility for working metal. Significantly, that facility is not possessed by the Australian aborigine. Stone meteorites, which had no practical use, were invariably worshipped. Moslem pilgrims to Mecca pay homage to the sacred black stone of Kaaba, and in Japan the Ogi meteorites were worshipped for 150 years in the belief that they were weights which had fallen from the loom of the Goddess Shokujo who lived on the shores of the Heavenly River (the Milky Way).

The problems of estimating the influx of meteorites and the ratio between stones and irons have been discussed by Nininger (Out of the Sky, an Introduction to Meteoritics, Dover New York; 1952) who searched for witnessed and unwitnessed meteorite falls for more than 30 years. Campaigns among rural populations in the United States were designed to acquaint farmers and ranch dwellers with the appearance and importance of meteorites. Directions were given for simple field tests to distinguish meteorites from terrestrial rocks and a price was offered for specimens as an inducement to all to keep on the lookout and report them. The programme was very successful: the number of finds increased dramatically and eventually the content of the Nininger Collection nearly mirrored the prevalence of meteorites in space (where irons make up $6 \%$ by number, stony-iron $2 \%$, and stones $92 \%$ ).

In Australia the intensive prospecting for gold and other minerals led people to expect a reasonable yield of meteorites. But, unfortunately, the prospectors avoided the flat, sandy, desert regions of the interior plains where meteorites would be more obvious. Stockmen and ploughmen may have found meteorites but, unlike the prospector, they generally had no interest in unusual rocks. On the Nullabor Plains, however, the personnel at the Kalgoorlie School of Mines have encouraged an active interest among the rabbit trappers. 